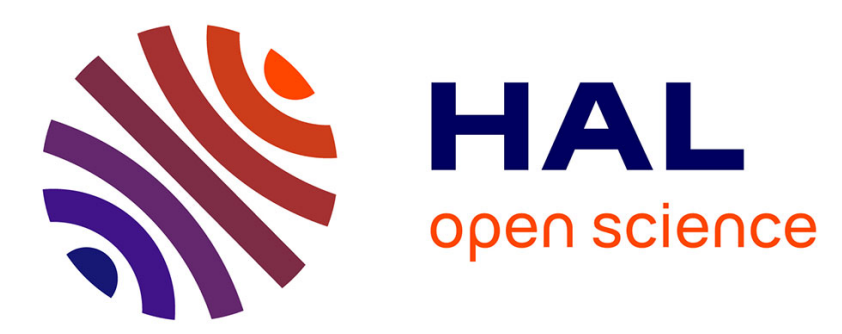

\title{
Un exemple d'application des datations radiocarbone à l'interprétation d'une stratigraphie complexe: la fouille des ateliers de Plussulien (Côtes-du-Nord)
}

Georgette Delibrias, Charles-Tanguy Le Roux

\section{- To cite this version:}

Georgette Delibrias, Charles-Tanguy Le Roux. Un exemple d'application des datations radiocarbone à l'interprétation d'une stratigraphie complexe: la fouille des ateliers de Plussulien (Côtes-du-Nord). Bulletin de la Société préhistorique française, 1975, 72 (3), pp.78-82. 10.3406/bspf.1975.8354 hal03407117

\section{HAL Id: hal-03407117 \\ https://hal.science/hal-03407117}

Submitted on 28 Oct 2021

HAL is a multi-disciplinary open access archive for the deposit and dissemination of scientific research documents, whether they are published or not. The documents may come from teaching and research institutions in France or abroad, or from public or private research centers.
L'archive ouverte pluridisciplinaire HAL, est destinée au dépôt et à la diffusion de documents scientifiques de niveau recherche, publiés ou non, émanant des établissements d'enseignement et de recherche français ou étrangers, des laboratoires publics ou privés. 


\title{
Un exemple d'application des datations radiocarbone à
} l'interprétation d'une stratigraphie complexe : la fouille des ateliers de Plussulien (Côtes-du-Nord)

\author{
Georgette Delibrias, Ch.-T. Le Roux
}

\section{Résumé}

Résumé. - Les ateliers de Plussulien, producteurs des haches polies en dolérite du Type A, ont fait l'objet, pendant leur fouille, d'une série de 19 datations radiocarbone. Les dates sont discutées ici en fonction de l'évidence stratigraphique, ce qui amène à proposer, pour le déroulement des trois grandes phases d'activité reconnues, une période s'étendant de 4000 à 2500 av. J.-C. environ, en dates calibrées.

\section{Citer ce document / Cite this document :}

Delibrias Georgette, Le Roux Ch.-T. Un exemple d'application des datations radiocarbone à l'interprétation d'une stratigraphie complexe : la fouille des ateliers de Plussulien (Côtes-du-Nord). In: Bulletin de la Société préhistorique française. Comptes rendus des séances mensuelles, tome $72, n^{\circ} 3,1975 . p p .78-82$;

doi : https://doi.org/10.3406/bspf.1975.8354

https://www.persee.fr/doc/bspf_0249-7638_1975_num_72_3_8354

Fichier pdf généré le 08/09/2020 


\title{
Un exemple d'application des datations radiocarbone à l'interprétation d'une stratigraphie complexe : la fouille des ateliers de Plussulien (Côtes-du-Nord)
}

\author{
par (i. Delibrias * el C.-T. Le Roux * *
}

\begin{abstract}
Résumé. -.. I.es ateliers de Plussulien, producteurs des haches polies en dolérite du Type $A$, ont fait l'objet, pendant leur fouille, d'une séric de 19 datations radiocarbone. Ies dates sont discutes ici en fonction de l'évidence stratigraphique, ce qui amène a proposer, pour le déroulement des trois grandes phases d'activité reconnues, une période s'étendant de 4000 à 2500 av. J.-C. environ, en dates calibrées.
\end{abstract}

Les ateliers de taille localisés par le second d'entre nous en 1964 à l'Est du village de Sélédin en Plussulien, dans le Sud du département des Còtes-du-Nord (1) représentent, rappelons-le, le site d'origine de toutes les haches polies en "Dolérite du Type A », roche définie par „. Cogné et P.-R. Giot dès 1952 comme constituant près de $40 \%$ de toutes les haches polies en roche dure de Bretagne (2). On en trouve également en abondance dans tout l'Ouest et de facon plus sporadique un peu partout en France, en Belgique et dans le Sud de l'Angleterre où cette roche correspond all "Group $X$ 》 des chercheurs britanniques ( 3 ).

Après des prospections et sondages préliminaires, cinq campagnes de fouilles ont été entreprises en l'un des points de ce vaste site depuis 1969 ; elles ont donné lieu à plusieurs publications provisoires (4) de sorte qu'il n'est pas

(*) Centre des Faibles Radio-activités C..E.A./C....R.S.

(*) Direction des Antiquités Préhistoriques de Bretagne, E.R. du C.N.R.S., n⿳ 27.

(1) LE Rovx C.-T. et Gıor P.-R. -- Etude pétrographique des haches polies de Bretagne, VII, Bull. Soc. Prehist. Fr., C.R.S.M. h2, 1965 , pp. CXXVill-C.XXIX.

(2) Cogsf J. ef Gior P.-R. ... Etude petrographique des haches polies de Bretagne, I, Bull. Soc. Préhist. Fr., 19, 19:2, pp. 338395.

(3) Evexs E. D., Surtu I. F. et Warios F. S. - The petrological identification of stone inplements from South-liestern England ; Fifth report of the Sub-Committee of the SouthII estern Federation of Museums and Art Galleries, Proc. Prehist. Soc., 38, 1972, pp. 233-275.

(4) a) Is Botx (..-T., in : "Chronique de Préhistoire et de Protohistoire 》 par P.-R. (iiot, Bull. et M:m. Soc. Emul. C.d..." nécessaire de revenir ici sur l'aspect purement archéologique de ces travaux. Par contre, nous voudrions insister sur les conditions particulières de ce chantier qui a révélé une stratigraphie complexe mais pratiquement dépourvue de tout «fossile directeur », l'évolution des techniques d'exploitation de la roche et de façonnage des objets ne variant pas assez nettement pour permettre de distinguer autre chose que de grandes phases dont aucun document archeologicue ne permettait d'ailleurs de situer nettement la position dans le temps, ni la durée réelle.

C'est une série exeeptionnelle de 19) dates radiocarbone fournies par la première d'entre nous qui est maintenant disponible, correspondant à des analyses effectuces au fur et à mesure de lavancement de la fouille (j). Ces datations, obtenues progressivement, campagne après campagne, ont souvent permis de vérifier et parfois de rectifier les interprétations faites sur le terrain ; leurs bandes d'incertitudes $( \pm 1$ or $)$ se recoupant souvent largement, elles ne sauraient, en elles-mèmes, fournir la base d'une chronologie détaillée. En confrontant les datations avec la lecture d'une stratigraphic complexe et parfois peu explicite, on peut cependant élaborer une séffuence assez précise, et surtout bien calcé chronologiquement. Ce travail, qui a été mené parallèlement à l'avancement de la fouille, a soulevé quelques problèmes qu'il nous a paru intéressant d'exposer en détail ici.

XCY, 1967, pp. 123-124 ; XCXIII, 1970, pp. 160-163 ; XC:IX, 1971.

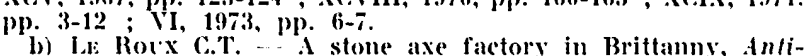
quily. X1., $1971, \mathrm{pp}$. $283-288$.

quily, Xlx, 1971,pl). $283-288$.

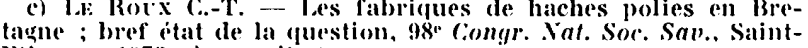
Etienne, 1973 (à paraitre).

(i) (itor P.-R. - Informations archeologiques, Gallia-Preihistoire $X, 1967, \mathrm{pp}, 334-335$; XII, 1969, p. 110; XIV, 1971, pp $312-34 ;$; Xi. 1973 (a paraitre).

(i) al (iorr l'.-R. … (Chronique des datations radiocarbone armoricaines. Annales de Bret., I.XXVII, 1970, pp. 1:36-1.57;

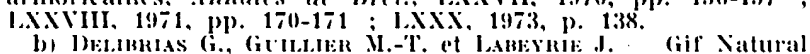
Radiocarbon Measurenents, Vili, Radiocarbon. 


\section{I. - STRATIGRAPHIE IDE LA COLPE PRIN- (IPAIE DU GISEMENT.}

La partic du site que nous avons étudiée reprósente un gros pointement rocheux, attaqué de toutes parts par les exploitations mais surtout à l'Est où une véritable carrière ouverte à sa base a été largement recomblée par les déchets de taille. 12 des dates obtenues concernent la coupe principale dégagée dans ce remplissage, épais de près de trois mótres.

La stratigraphie révéléce est fort complexe ; plutôt qu'une suceession de couches, il s'agit d'un empilement irrégulier de lentilles de natures variées : poches terreuses, lits de petits éclats, poches de gros bloes ou amas de cendres et de charbons de bois, avec tous les mélanges et intermédiaires possibles, mais aussi avec des remaniements ef surcreusements. D'une manière très générale cependant, on peut noter un pendage grossièrement dirige vers le pied du rocher ce qui correspond à la tendance naturelle des ouvriers à déchausser au maximum la roche et à rejeter leurs déblais en arrière à faible distance.

Cette accumulation se subdivise assez nettement en quatre " phases » séparces par des niveaux d'arrêt asse\% précis que l'on peut appeler « sols » par commodité, mème s'ils n'ont le plus souvent aucune réalité pédologique.

Phase I : Niveaux essentiellement terreux, avec petits foyers et lits de charbons de bois dispersés, formant un important tas conique dans la partie Est de la coupe, se réduisant à un mince placage discontinu sur le rocher dans la partie centrale et disparaissant dans la partic Ouest. Sa surface constitue le "Sol 4 ». Cette formation repose sur le rocher frais ou altéré, directement ou par l'intermédiaire d'un lit d'argile grise (Sol 5).

Phase II : Sćrie beaucoup plus pauvre en matériaux terreux, constituée essentiellement de bloes et d'éclats souvent à sec, dans lesquels sont cmballés de nombreuses pièces brisées ou inachevées et aussi d'énormes blocs de plusieurs tonnes réutilisés comme établis de taille.

Phase III : Série analogue à la précédente, dont elle est séparce par un important niveau d'arrêt nettement humifié (Sol 3). Il n'y a plus de gros bloes-établis mais on note d'énormes foyers placpués contre le rocher et visiblement destinés à l'abattage de la pierre. Cette série se termine par un mince lit de petits éclats formant une légère discontinuilé dans la stratigraphice (Sol 2).

Phase IV : Correspond a des éboulements et remaniements des niveaux superficiels après l'arrèt de l'activité du site, donnant un mélange de blocs, d'éclats et de terre, humifié par le sol actuel (Sol 1).

La fouille a d'abord altaqué les niveaux correspondant à la phase IV, peu épais et surtout développés en pied de falaise. Un décapage minutieux a pu mettre en évidence plusieurs petits niveaux d'arrèt sub-horizontaux se traduisant simplement par une discontinuité dans l'aceumulation des matériaux par ailleurs assez disparates et mòlés de grains de charbons de bois dispersés. Il était done permis de se demander si l'on avait une formation en place ou non. La datation obtenue pour les charbons recueillis (GIF 1538: $4550 \pm 140$, soit 2600 B.C.), qui semble fort correcte par elle-mème, démontre en fait une contamination par des éboulements depuis le sommet du rocher quand on la compare avec le resultat des autres analyses.

La puissante série de la phase III était caractérisée, nous lavons vu, par l'abondance des déchets d'exploitation de tous calibres el la présence de grands foyers d'abattage de la roche. La datation de l'un de ces brasiers parfaitement en place (GIF : 1539 : $4050 \pm 130$, soit 2100 B.C..) permet de situer cette phase au Nélithicque Final et implique du mème coup que la date plus ancienne fournie par les niveaux sus-jacents soit due à une perturbation par addition de charbons anciens.

L'explication devient claire quand on sait que le sommet du rocher au pied ducpuel la coupe a ité relevée porte lui-même des traces d'exploitation assez importantes, mais relativement peu de déchets de taille encore en place, ceux-ci n'ćtant guère conservés que dans les anfractuosités par suite de la pente. Dans l'une de ces poches, un petit foyer a pu ètre daté, d'une part à partir de grains de charbons dispersés (GIF $1542: 4930 \pm 130$, soit 2980 B.C.) et d'autre part à partir du foyer lui-même, trouvé bien en place l'année suivante (GIF 1871: $4950 \pm 140$, soit 3000 B.C.). Ces deux dates anciennes ef parfaitement cohérentes situent donc avec précision l'exploitation du sommet du rocher et peuvent expliquer la présence de grains de charbons anormalement anciens dans les niveaux supérieurs de la carrière située en contrebas, à la suite de chutes de matériaux ou mème de simples lessivages par la pluie.

En dessous du Sol 3, la fouille de l'ensemble complexe constituant la «Phase II " a posé des problèmes d'interprétation délicats qui n'ont trouvé leur solution que progressivement.

A la fouille, on a pu mettre en évidence plusieurs cas de surcreusements el de remaniements anciens, souvent à peine discernables par suite de la nature très pierreuse du milieu. La position stratigraphique de ces recreusements reste mal assurée, tout au plus peut-on affirmer que le principal d'entre eux recoupait le Sol 3 et lui était done postérieur, appartenant vraisemblablement à la Phase III. La stratification de cette zone était également complicuée par la présence de plusieurs blocs géants, de plusieurs tonnes chacun, généralement utilisés comme établis. Tous étaient recouverts par le «Sol 3 » et leur appartenance à la Phase II ne fait done bas de doute ; l'un d'entre cux (bloc A) était calé dans une fosse creusée a travers l'accumulation des éclats jusqu'au "Sol 4 » tandis que les autres 
flotlaient parmi le remplissage tout en étant semble-t-il soigneusement mis en place (en particulier le bloc B était parfaitement juxtaposé au bloc $A$ pour former avec lui un large établi).

Trois échantillons prélevés au voisinage immédiat des blocs $A$ et $B$ ont donné une série de dates bien homogènes :

(GIF 1872 : $5100 \pm 140$, soit 3150 B.C. pour un foyer situe au voisinage immédiat du sommet de $A$, mais apparemment relić all Sol 3 .

GIF $1873: 5150 \pm 140$, soit 3200 B.C. pour des charbons prélevés parmi les petits éclats encore encroûtés sur le sommet martelé du Bloc $\mathrm{B}$.

GIF 1874 : $5075 \pm 140$, soit 3125 B.C. pour une lentille de terre charbonneuse coincée entre les bases des deux blocs $A$ et $B$.

En fait ces trois dates apparaissent plutòt hautes si on les compare aux quatre autres, obtenues pour cette même phase II et qui constituent elles aussi une série cohérente :

GIF $2328: 4790 \pm 110$, soit 2840 B.C. pour un foyer situé a la base de la Phase II, juste au-dessus du «Sol 4 ».

GIF $1540: 4700 \pm 150$, soit 2750 B.C. pour un petit foyer plaqué contre le rocher, sous le «Sol 3 ».

GIF 1541 : $4500 \pm 130$, soit 2550 pour un foyer situé près de la base du bloc $A$.

GIF $2683: 4350 \pm 115$, soit 2400 B.C. pour le petit foyer trouvé dans la fosse de calage du bloc $A$.

levant ces deux sćries de dates a priori aussi «bonnes» les unes que les autres, mais néanmoins difficilement compatibles, on peut formuler deux hypothèses :

1" Les blocs $A$ et $B$ ont bien été mis en place avant - 3100 et la date GIF 1540 indiquerait la fin de la Phase II en même temps que le début de la formation du Sol 3 qui correspondrait à un très long arrêt, ce qui expliquerait qu'il ait eu le temps de s'humifier notablement avant la Phase III. Cela implique par contre que les autres datations de la Phase II soient malencontreusement tombées à la limite de leur bande d'incertitude $\pm 1_{\sigma}$, ou que les échantillons correspondent à des mélanges. Ce n'est pas impossible pour GIF 1541, situć assez près en contrebas des grands foyers de la Phase III ; c'est beaucoup plus improbable pour GIF 2328, éloigné de toute source de contamination visible, et pour GIF 2683, scellé par le bloc A lui-même. Ce dernier échantillon forme d'ailleurs un doublet avec GIF 1541, ces prélèvements très voisins ayant donne des dates assez proches pour que leurs marges d'erreur se recouvrent largement, ce qui les confirme l'une à l'autre; il y a de fortes chances pour que les charbons des deux échantillons aient en fait la mème origine.

On peut penser au contraire que GIF 2328 est bien correct pour le début de la Phase II et que la mise en place des blocs $A$ et $B$ soit assez tardive, faite a la faveur d'un profond recreusement (reconnu en fouille et daté par (iIF $15+1$ et GIF 2683) ayant respecté un lambeau de couche plaquée contre le rocher et contenant le foyer (iIF 1540 .

Mais comment expliquer les trois dates trop anciennes (de plus de 500 ans) obtenues au voisinage des bloes $A$ et $B$ ? On peut éliminer l'erreur de manipulation car ces trois échantillons voisins ont domne des résultats suffisamment homogènes pour qu'on puisse les considérer comme provenant en fait du mème bois et done se confirmant les uns les autres, la dispersion des fragments ayant pu se faire au moment mème de la combustion ou peu après (on peut noter que les échantillons (iIF 1872 et GIF 1873, parfaitement circonscrits et délimités, itaient très voisins, tandis que GIF 1874, plus diffus, se trouvait en contrebas des précédents). On est donc amené à faire intervenir l'âge du bois, mais 500 ans est un vieillissement un peu fort pour qu'on puisse l'expliquer par la seule utilisation d'un coeur d'arbre mort, fùt-il multicentenaire. Quant à la réulilisation comme combustible de vieux bois d'ceuvre pris dans des constructions anciennes, cela nous obligerait, à remonter à une époque antérieure à l'ouverture de l'atelier, comme nous le verrons plus loin. D'autre part, l'hypothèse d'un remaniement de charbons anciens associés à la phase I lors de la mise en place des bloes $A$ et $B$ est pratiquement incompatible avec les observations faites en fouille.

Quoiqu'il en soit, cette seconde hypothèse semble confirmée par les datations obtenues pour la Phase I, qui a donné une série particulièrement bien groupée :

$$
\begin{aligned}
& \text { GIF } 1875: 4960 \pm 140 \text {, soit } 3010 \text { B.C. } \\
& \text { GIF } 2329: 4960 \pm 110 \text {, soit } 3010 \text { B.C. } \\
& \text { GIF } 2330: 4960 \pm 110 \text {, soit } 3010 \text { B.C. } \\
& \text { GIF } 2682: 4940 \pm 110 \text {, soit } 2990 \text { B.C. }
\end{aligned}
$$

Ces dates ont été obtenues à partir de foyers situés à la base de la formation, contre le rocher ou sur le «Sol 5 » (GIF 2330 et GIF 2682) ou par des petits amas de charbons recueillis en plein remplissage dans des zones particulièrement intéressantes, en association avec un petit outillage fruste en silex (GIF 1875) ou de petits tessons de céramicjue malheureusement non identifiables (GIF 2329). Cela implique que cette Phase I ait eu une durée assez brève, malgré l'épaisseur des couches correspondantes, ce qui est à rapprocher de la date GIF 2328 obtenue pour le début de la Phase II.

En dehors de cette coupe principale, nous avons déja ru les dates obtenues pour l'exploitation du sommet du rocher ; elles permettent de paralléliser cette partie du gisement avec la Phase I de façon catégorique, ce qui aurait été bien difficile à affirmer par la seule analyse typologique de l'industrie, peu abondante d’ailleurs dans cette zone. 
En pied de falaise, mais un peu plus au Sud. une autre zone de fouille a livré, a sa base, de nombreux charbons rassemblés dans l'argile correspondant a notre «Sol 5 »; la date obtenue (GIF 1876 : 5160) \pm 140, soit 3200 B.(.) est un peu plus ancienne que celles de la Phase I, en position straligraphique équivalente dans la coupe principale ; on peut la aussi envisager l'ulilisation d'un bois âgé (une centaine d'années suffirait pour expliquer le décalage en permettant aux bandes d'incertitude \pm 1 o de se chevalucher eonfortablement) ou bien admettre le démarrage un peu plus précoce de lexploitation dans cetle zone, ce qui n'a rien d'impossible.

Plus loin vers l'Est, a bonne distance de l'aflleurement, un large décapage nous a permis de dégager un très beau « sol " correspondant a un atelier de taille et de retouche, pratiquement sans trace de gros débitage, avec deux gros blocs-établis bien en place et une curieuse petite fosse ovalaire sommairement parée à l'aide de bloes de taille moyenne ; son remplissage éait formé d'un mélange de terre cendreuse, malheureusement très pauvre en grains de charbon de hois, et de petits éclats de retouche. Par contre, d'autres poches cendreuses moins nettement détimilées ont íté reconnues au voisinage, reposant sur le mème sol et l'une d'elles a pu fournir assez de charbon pour donner une datation (GIF 1877 : 5270 \pm 140, soit 3320 B.C..). C'est un résultat qui, au moment où nous l'avons obtenu, nous a paru confirmer la date ancienne des blocs-établis $A$ et $B$; mais avec la suite de la fouille, le sol de cet atelier a pu ètre très nettement raceordé au «Sol 4 » de la coupe principale, tandis que les niveaux de la Phase I étaient effectivement rencontrés au-dessous. Force est donc d'envisager, ici encore, une cause de vieillissement, quelle qu'elle soit, mais sans doute la mime que pour GIF 1872, GIF 1873 et GIF 1874.

Enfin, plus bas sur la pente, une dernière date a été obtenue pour des grains de charbon dispersís dans un niveau malheureusement soliflue ; le risultat (GIF 1543 : 4360$) \pm 130$, soit 2410 B.P.) peut s'interpréter, soil par un mélange de grains d’âges variés (Phases II et III par exemple), soit comme âge réel d'un échantillon homogène qui serait alors contemporain de la fin de la Phase II. Cette dernière hypothèse concorderait assez bien avec l'idée d'une intense activité régnant alors sur le site comme en témoignent l'importance des couches de ce slade et la dispersion des blocs-établis ; dans ces conditions on peut considérer comme normal qu'une bonne part des sites de taille établis à distance de l'affleurement correspondent à cette phase de prospérité.

\section{CONCLLISIONS.}

Des quatre grandes phases qui seules apparaissaient clairement à la fouille, l'utilisation systimatique des datations radiocarbone a permis de dégager une histoire cohérente du site et de situer son diroulement dans le temps aree une bonne précision, en l'absence de tout autre élément de datation de valeur comparable. Si nous essavons de « calibrer » les dates obtenues en années calendaires nous pouvons résumer l'histoire des ateliers de la facon suivante (6) :

Dès 3800 (peut-itre avant - $4000 \%$ ), décollverte du gisement qui est décapé (lambeaux du Sol i) of intensément exploité (Phase I) dés 3600 - 3500, mais essentiellement dans les zones fracturées où les bloes de roche saine, emballés dans une gangue d'argile d'altération, sont relativement faciles à dégager selon des technique's asse\% proches de celles utilisées dans les exploitations de silex où les rognons sont pris dans la craie tendre.

Cette premiere phase d'activite correspond a lapparition des haches polies du "Type $A$ » dans léquipement des populations du Néolithique moven régional, après une période qui a, semblet-il, ignoré ce matériau, qu'on ne trouve jamais, en particulier, dans les dolmens à couloir les plus anciens.

Parallèlement, ont lieu les premières tentatives d'exploitation de la roche massive par percussion des parties déjà fortement fissurées par les diaclases, sur le sommet de l'affleurement en particulier, où l'allération météorique avait dû préparer le terrain, en quelque sorte.

Bientot, peut-ètre par suite de l'épuisement des zones favorables à la récupération de blocs dans l'argile, cette seconde technigue se généralise, sans doute a partir de - 3500 environ (Sol 4), el se développe sur une vaste échelle ; les ouvriers, sans doute familiers des techniques de manutention de l'architecture mégalithique, n'hésitent pas à déplacer des blocs de plusieurs tonnes pour multiplier les établis de taille et de retouche autour de la zone d'extraction.

I'n abandon prolongé parait se produire, an moins localement, peu après -- 3000, laissant à la végétation le temps de reprendre possession des lieux et de former le Sol 3.

Après cet arrèt, l'activité reprend au Néolithique final avec une innovation technique, l'utilisation du feu pour l'abatlage en grand de la roche et les ateliers sont à nouveau en pleine prospérite vers 2000. Enfin, une activite réduite a pu se maintenir quelque temps, sans doute tuée progressivement par l'abondance de plus en plus grande des premiers objets de métal. Bien qu'aucun niveau non perturbé de cette période n'ait été retrouré, quelques haches bipennes ou haches marteaux en "Dolérite $A$ " sont connus en collection; ils peuvent appartenir a cette phase lardive comme semblerait le montrer une ébauche briscéc de hache bipenne récolté dans les niveaux superficiels de la fouille.

(6) I.e callibrage des dates a éte latit en se basant sur la conrbe commode de :

Switsin V. R. -... The madiocarbon recalibrated, Antiquity, XiNII. 1973, pp. 131-137. 


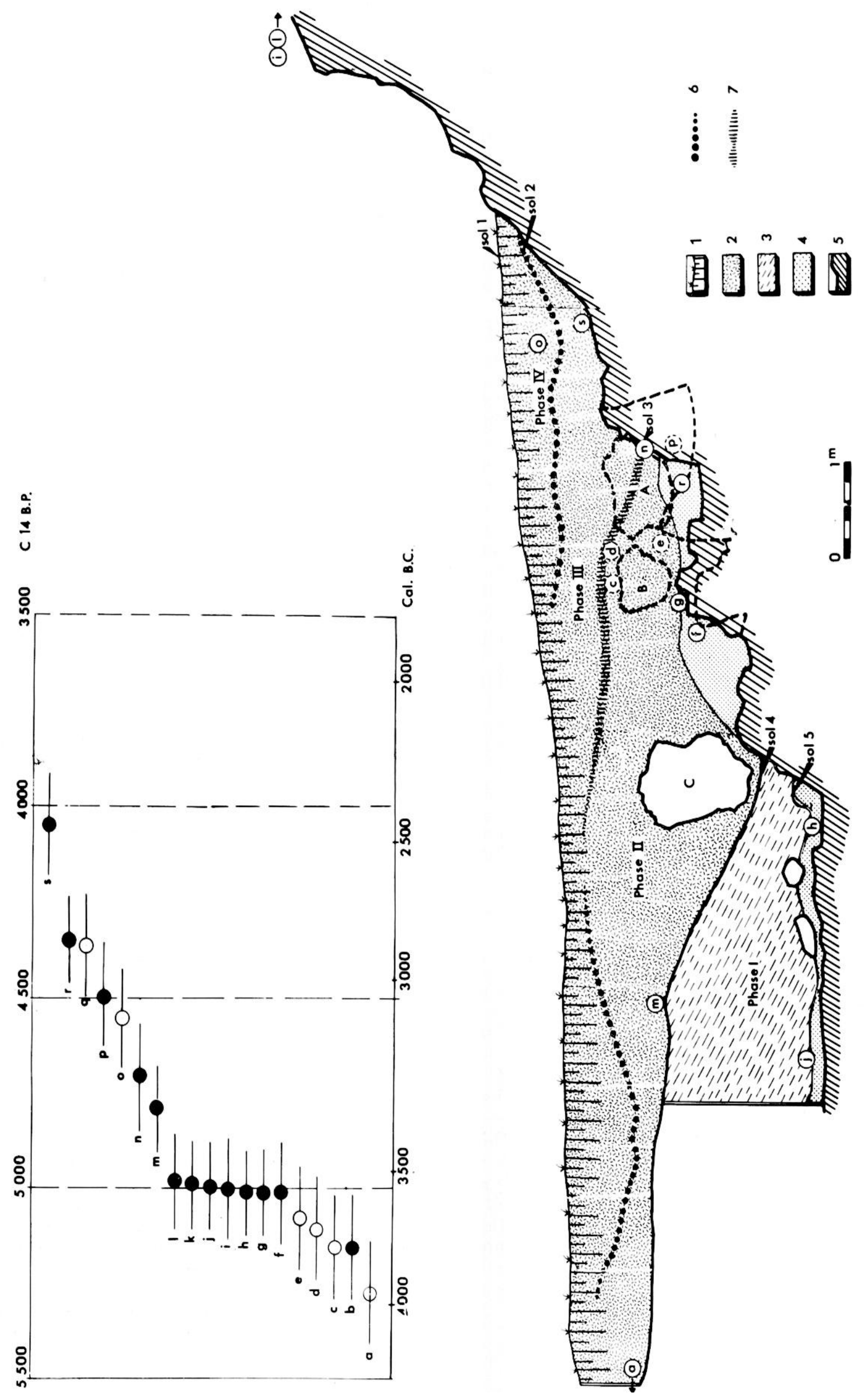

lig. 1. PldStlitex, Ateliers de Seledin, coupe principale simplitie ten pointilles, projections sur la coupe depuis un plan situe a colviron $2 \mathrm{~m}$ en aviant).

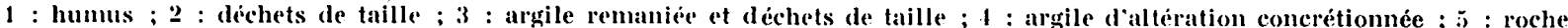
saine en place ; 6 : niveau d'arrèt : 7 : horizon humifiee. $A, B, C:$ Blocs-établis ; (a) à (s) : emplacements des echantillons dates.

En cartouche, tableau synthétique des dates obtenues (en cercles blancs, celles dont rinterprétation pose des problèmes

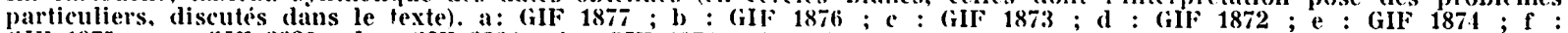

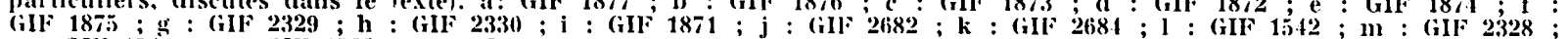

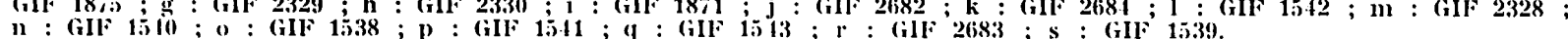

